\title{
Induced IGF-1R activation contributes to gefitinib resistance following combined treatment with paclitaxel, cisplatin and gefitinib in A549 lung cancer cells
}

\author{
XIAOJUN GE ${ }^{1 *}$, QINGJUAN CHEN ${ }^{2 *}$, YANG PING WU ${ }^{1}$, YUCHEN ZHANG ${ }^{1}$, HONGWEI XIA ${ }^{1}$, DANDAN YUAN ${ }^{2}$, \\ QI CHEN ${ }^{2}$, WEIBING LENG $^{2}$, LIANG CHEN ${ }^{1}$, QIULIN TANG ${ }^{1}$, XIAOHUI PANG ${ }^{2}$ and FENG BI $^{2}$ \\ ${ }^{1}$ Laboratory of Signal Transduction and Molecular Targeted Therapy, State Key Laboratory of Biotherapy, and \\ ${ }^{2}$ Department of Medical Oncology, West China Hospital, Sichuan University, Chengdu, Sichuan 610041, P.R. China
}

Received March 18, 2014; Accepted May 19, 2014

DOI: 10.3892/or.2014.3331

\begin{abstract}
Gefitinib demonstrates excellent performance in the treatment of lung adenocarcinoma patients; yet, there was no added benefit in combination with chemotherapy as reported in a phase III clinical trial. For exploring the mechanism of the failed combination therapy in lung cancer, in the present study, four therapy assessment groups, including a control group, a chemotherapy group [paclitaxel + cisplatin (TP)], a gefitinib monotherapy group $(\mathrm{G})$ and a combination group [paclitaxel + cisplatin + gefitinib $(\mathrm{TP}+\mathrm{G})]$, were established in an A549 cell line and mouse xenotransplanted tumor models. By HPLC, we found that the gefitinib concentration was significantly higher in the combination group when compared to that in the $\mathrm{G}$ group in the non-small cell lung cancer cell line, A549 $(\mathrm{p}<0.05)$. Following the treatment time extension, an increased cell growth rate was observed in the combination group, while the cellular concentration of gefitinib was not decreased. The expression levels of P-IGF-1R, P-SRC and P-ERK in the fourth combination treatment group were significantly higher than levels in the fourth $G$ treatment and control groups $(\mathrm{p}<0.05)$. Following downregulating of IGF-1R
\end{abstract}

Correspondence to: Dr Feng Bi, Laboratory of Signal Transduction and Molecular Targeted Therapy/Department of Medical Oncology, West China Hospital, Sichuan University, Chengdu, Sichuan 610041, P.R. China

E-mail: bifeng@medmail.com.cn

*Contributed equally

Abbreviations: EGF, epidermal growth factor; EGFR, epidermal growth factor receptor; IGF-1R, insulin-like growth factor receptor 1; G, gefitinib monotherapy; TP, paclitaxel + cisplatin; $\mathrm{TP}+\mathrm{G}$, paclitaxel + cisplatin + gefitinib; G4, the fourth gefitinib treatment; $(T P+G) 4$, the fourth TP and gefitinib treatment; HPLC, high performance liquid chromatography

Key words: chemotherapy, gefitinib, lung cancer, drug resistance, IGF-1R in the fourth combination treatment group, drug sensitivity was recovered in vitro. In the mouse model, compared with the gefitinib monotherapy group, the combination group exhibited a smaller tumor volume, lower body weight and reduced survival rate $(\mathrm{p}<0.05)$. Gefitinib concentrations in the serum and tumor tissues in the combination therapy group were also decreased when compared with these concentrations in the gefitinib alone group. The present study is the first to demonstrate that the decreased gefitinib concentration in serum and tumor tissues is one of the reasons resulting in the failed combination treatment (chemotherapy + gefitinib) in vivo study. Frequent use of the combination treatment in A549 lung cancer cells induced IGF-1R activation which contributed to gefitinib resistance and gave rise to the failure of the combination therapy.

\section{Introduction}

Lung cancer still remains the leading cause of cancer-related mortality in the world, and the majority of patients present with advanced disease at diagnosis or develop recurrence, neither of which is amenable to curative approaches (1). The epidermal growth factor receptor (EGFR) is expressed in a number of tumors, including non-small-cell lung cancer (NSCLC). EGFR is a member of the EGFR family that includes HER2 (ErbB2), HER3 (ErbB3) and HER4 (ErbB4) (2-4). Activation of the EGFR leads to receptor associated tyrosine kinase activity that initiates a cascade of events leading to downstream signaling and a variety of changes characteristic of malignant progression including upregulation of ras, raf, mitogen-activated phosphorylated (MAP) kinase, and phosphatidylinositol 3-kinase (PI3K) and the downstream protein-serine/threonine kinase Akt. In turn, cellular growth and invasive capacity are enhanced. Preclinical data indicate that small molecules that compete with adenosine triphosphate for delivery of phosphate groups to critical tyrosine residues could block signal transduction through EGFR. Studies have demonstrated that gefitinib is a promising agent for the treatment of a wide range of tumors, including NSCLC. Gefitinib can enter cells and inhibit the autophosphorylation of EGF-stimulated EGFR in a variety of EGFR-expressing human cancer cell lines (5). Several 
phase I studies have shown that gefitinib is generally well tolerated, with evidence of antitumor activity in NSCLC, and two large phase II gefitinib monotherapy studies [Iressa Dose Evaluation in Advanced Lung Cancer (IDEAL) 1 and 2] in patients with pretreated advanced NSCLC further confirmed that gefitinib was generally well tolerated and demonstrated clinically significant antitumor activity (6-8). Cetuximab is an IgG1 subclass chimeric mouse-human antibody that plays roles similar to gefitinib. In addition, cetuximab binds to the extracellular portion of EGFR and induces destruction of the receptor (9). Addition of cetuximab to platinum-based chemotherapy results in a moderate increase in the overall survival of patients with advanced NSCLC as confirmed in clinical research (10). There is a strong rationale for combining gefitinib with standard chemotherapeutic agents. Unfortunately, this exciting, high-level of preclinical activity has not transpired in most clinical studies reported to date. In fact, the addition of gefitinib has offered minimal to no additive or synergistic clinical activity to the standard antineoplastic regimens which has led to its limited availability (11-15). Likewise, two phase III trials have not shown gefitinib to be effective when combined with chemotherapy in the treatment of NSCLC $(16,17)$. Paclitaxel + cisplatin (TP) chemotherapy regimens are used for the treatment of NSCLC (18). We hypothesized that one of the reasons resulting in the failure of combination therapy was that chemotherapy drugs affect the cellular gefitinib concentration. Thus, the present study investigated the effects of combining gefitinib with TP on growth factor signaling, cell cycle progression, and apoptosis in vitro and in vivo. Subsequently, we evaluated the difference in cellular gefitinib concentration after gefitinib monotherapy and after combination therapy with gefitinib and TP to confirm our hypothesis.

\section{Materials and methods}

Chemical preparation. Gefitinib was purchased from AstraZeneca (Macclesfield, UK). A $10 \mathrm{mM}$ working solution in DMSO was prepared and stored at $-20^{\circ} \mathrm{C}$. Recombinant human EGF was purchased from PeproTech (London, UK). Fetal bovine serum (FBS) was purchased from Gibco Invitrogen Co. (Carlsbad, CA, USA). The primary antibodies used for western blot assays were as follows: rabbit monoclonal for anti-EGFR antibody, anti-phospho-EGFR (PY1068) antibody, anti-IGF1R antibody, anti-phospho-IGFR (Tyr1135/1136) antibody, anti-SRC antibody, anti-phospho-SRC (Tyr416) antibody, anti-ABCG2 antibody, anti-ERK1/2 antibody, anti-phosphoERK (PY204) antibody, anti-Akt antibody, anti-phospho-AKT (PS473) antibody (all from Abcam Co.); and mouse polyclonal anti-P53 antibody, anti-BAX antibody (from Cell Signaling Technology).

Cell culture. The lung adenocarcinoma A549 cell line was maintained in RPMI-1640 medium with $10 \% \mathrm{FBS}$ at $37^{\circ} \mathrm{C}$ in a $5 \% \mathrm{CO}_{2}$ atmosphere. For this study, we divided the A549 cells into four groups: group one remain in normal growth; group-two $(\mathrm{G})$ was administered gefitinib monotherapy and TP was added to group-three; group-four was administered the combination of gefitinib and TP. Group two, three and four received four repeated doses to simulate clinical situations.
Detection of gefitinib concentration in NSCLC A549 cells by high performance liquid chromatography (HPLC). The cells were cultured according to the experimental design and harvested. By thawing and refreezing five times in liquid nitrogen, the A549 cells were destroyed, added to a mixture of mobile phase methanol:water $(70: 30,500 \mu \mathrm{l})$, and the supernatant was directly injected into HPLC. The objective of this study was to compare the differences in the cellular gefitinb concentration between the $\mathrm{G}$ and $\mathrm{TP}+\mathrm{G}$ groups. The gefitinib concentration of one cell was equal to the concentration obtained by HPLC/total cell number. Assay performance was monitored using quality control samples, and the limit of quantification for each analysis was $0.05,0.1$ and $0.2 \mu \mathrm{M}$ for gefitinib. The linear relationship between the peak area and the concentration of gefitinib was good; it was convenient for calculation and comparison with the intracellular gefitinb concentration.

Cell growth inhibition assay. CCK-8 assays were used to evaluate the growth inhibitory effects of gefitinib, paclitaxel and cisplatin. The cells were seeded on 96-well plates at a density of 3,000 cells/well, incubated for $24 \mathrm{~h}$, and then treated for $48 \mathrm{~h}$ with the drugs at $37^{\circ} \mathrm{C}$. After drug treatment, CCK-8 solution was added to each well and incubated for $4 \mathrm{~h}$ at $37^{\circ} \mathrm{C}$. Cell viability was determined by measuring the absorbance at $540 \mathrm{~nm}$ in a microplate reader (micro-ELISA). Six replicate wells were used for each analysis, and at least three independent experiments were conducted. The data from the replicate wells are presented as the mean numbers of remaining cells, with $95 \%$ confidence intervals. To determine the effects of the combined drug treatments, any potentiation was estimated by multiplying the percentage of remaining cells (percent growth) for each drug.

Growth curves. Culture media were replaced every $48 \mathrm{~h}$ following drug treatment. The cells in the $\mathrm{G}$ and $\mathrm{TP}+\mathrm{G}$ groups were cultured for 5-10 days to allow cells to return to the logarithmic phase. Then cells were harvested and seeded on 96-well plates at a density of 800 cells/well. Three replicate wells were used for each analysis. Every three wells were select from two groups of cells on day 1, 2, 3, 4 and 5. CCK-8 solution was added to each well and incubated for $4 \mathrm{~h}$ at $37^{\circ} \mathrm{C}$. Cell viability was determined by measuring the absorbance at $540 \mathrm{~nm}$ in a microplate reader (micro-ELISA).

Analysis of the cell cycle and apoptosis. The cells were exposed to the different drug treatments for 48-72 $\mathrm{h}$ and were harvested. The cells were then washed with PBS and stained by PI at $4^{\circ} \mathrm{C}$ for 30 min using a cell cycle detection kit (KeyGen, Nanjin, China) and analyzed using a flow cytometer (BD FACSCalibur). Cells were stained using Annexin V-FITC and PI. Briefly, cells were suspended in binding buffer, followed by incubation with 1X Annexin V-FITC binding buffer (195 $\mu \mathrm{l})$ and Annexin V-FITC (5 $\mu \mathrm{l})$ for $10 \mathrm{~min}$ at room temperature in the dark. Expression of Annexin V and PI was determined using a fluorescence-activated cell sorting (FACS) flow cytometer (BD FACSCalibur). Apoptosis in the A549 cells was analyzed by Hoechst 33258 staining according to the manufacturer's instructions (Beyotime). Stained nuclei were observed under a fluorescence microscope. 
$A_{\text {mAl }}$

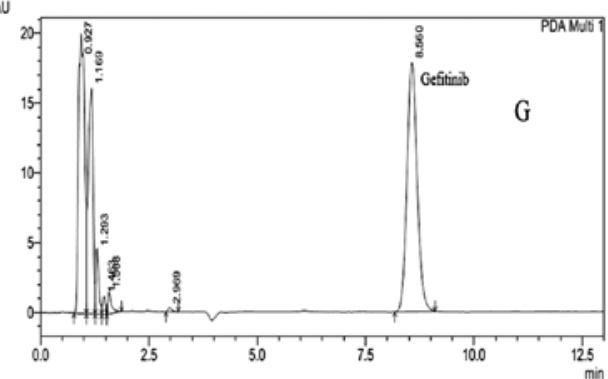

B

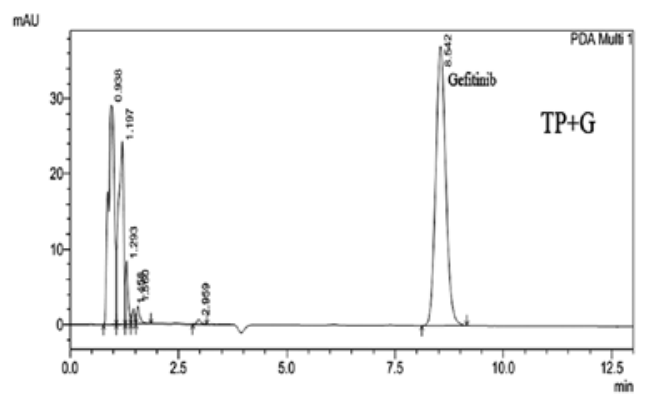

$\mathrm{C}_{\text {mal }}$

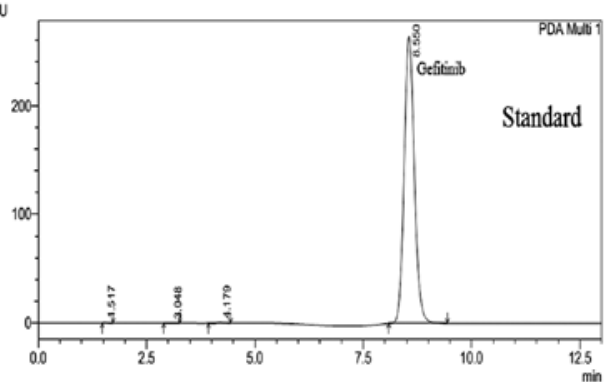

D

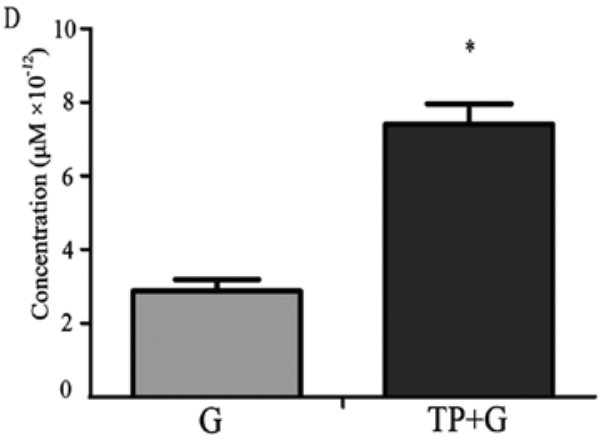

Figure 1. Cellular gefitinib concentration is significantly increased in the combination group. The data represent means \pm SEM from 3 independent experiments performed in triplicate. (A-C) Gefitinib chromatographic profiles in the G group, TP+G group, and standard (gefitinib; $0.1 \mu \mathrm{M}$ ) chromatographic profiles $\mathrm{x}$-axis is retention time (min); $\mathrm{y}$-axis is intensity (arbitrary units). The gefitinib concentration of one cell was equal to the concentration obtained by HPLC/total cell number. (D) Gefitinib concentration in the NSCLC A549 cells was significantly higher in the TP+G group than that in the G group. "p $<0.05$, compared with the G group.

Mutational analysis of the EGFR (exon 18-21) gene. Genomic DNA was extracted from the A549 cells. Primers were designed using Primer 5, and the sequences were as follows: 18 exon forward, 5'-GATGCCAAAGAAGTAGAATGAGA AAA-3' and reverse, 5'-CAGGAAAGCATCTTCACCCA CAG-3'; 19 exon forward, 5'-ATGGAATCTGTCAGCAAC CTCACCCTT-3' and reverse, 5'-CAACTGCTAATGGCCCG TTCTCG-3'; 20 exon forward, 5'-AACATGGTGAGGGC TGAGGTGAC-3' and reverse, 5'-ATGCCTTTGGTCTGTGA ATTGGT-3'; 21 exon forward, 5'-AAATTACTATGCAGCA TGTGGCACC-3' and reverse, 5'-TAGGATGTGGAGATGAG CAGGGT-3'. All mutations were confirmed at least twice from independent PCR isolates.

Western blotting. A549 cells and tumor samples were lysed in RIPA buffer (150 mM NaCl, 1\% NP-40, $50 \mathrm{mM}$ Tris-HCl $\mathrm{pH} 7.4,1 \mathrm{mM}$ phenylmethylsulfonyl fluoride, $1 \mu \mathrm{g} / \mathrm{ml}$ leupeptin, $1 \mathrm{mM}$ deoxycholic acid and $1 \mathrm{mM}$ EDTA) containing a cocktail of protease inhibitors and phosphatase inhibitors (Calbiochem, Darmstadt, Germany). Equal amounts of the protein samples (30-50 $\mu \mathrm{g}$ ) were separated by $12 \%$ SDS-PAGE and transferred to PVDF membranes (Millipore, Bedford, MA, USA) using the Bio-Rad Semi-Dry Transfer system.

Knockdown of IGF-1R by RNA interference and inhibitor for $S R C$. siRNA (sense, 5'-GAAGAGGUAUUGAAUGCUAdT dT-3' and antisense, 5'-UAGCAUUCAAUACCUCUUCT dTd-3') against IGF-1R was purchased from Guangzhou RiboBio Co., Ltd. Cells were transfected with short interfering RNAs at a final concentration of $40 \mathrm{nmol} / \mathrm{l} \mathrm{using}$ Lipofectamine 2000 (Invitrogen) in accordance with the manufacturer's instructions. Cell lysates were harvested $48 \mathrm{~h}$ after transfection. The SRC inhibitor, saratinib (5 $\mathrm{nM}$ ) was added to the culture medium for $48 \mathrm{~h}$, and the cell lysates were collected.

In vivo studies. Male athymic nude mice (4- to 6-weeks old) were purchased from Beijing HFK Bioscience Co., Ltd. Gefitinib was suspended at $50 \mathrm{mg} / \mathrm{kg}$ in $1 \%$ Tween-80 for administration by oral gavage. A549 cells were injected subcutaneously into the right dorsal flank of the mice. Tumor volumes were calculated using the formula: $\mathrm{Width}^{2} \mathrm{x}$ length $\mathrm{x} 0.52$. Tumor growth was monitored and when tumors reached an average of $200 \mathrm{~mm}^{3}, 40$ mice (injected with A549 cells) were randomized into 4 groups (10 mice/group). One group of female nude mice (control group) received $1 \%$ Tween- 80 alone $(0.2 \mathrm{ml}$ volume delivered as an oral gavage). The second group (bearing A549 tumor xenografts) was dosed orally with gefitinib at a dose level of $50 \mathrm{mg} / \mathrm{kg}$. The third group received paclitaxel (injected in the abdominal cavity at a dose level of $5 \mathrm{mg} / \mathrm{kg}$ ) and cisplatin (injected in the caudal vein at a dose level of $2 \mathrm{mg} / \mathrm{kg}$ ). The fourth group received gefitinib and TP (cisplatin and paclitaxel). Treatment was performed once daily. Tumor size and mouse weight were measured at the same time. After 14 days, the mice were sacrificed and the tumors were dissected. All animals were treated in accordance with National Institutes of Health guidelines for animal care and use, and experimental protocols were approved by the Institutional Animal Care and Treatment Committee of Sichuan University.

Determination of gefitinib concentration in the tumor samples. Twenty mice were sacrificed by inhalation of halothane at $2 \mathrm{~h}$ after the final dose, and blood samples were collected into heparinized tubes. The tumor samples were flash-frozen and 
stored at $-70^{\circ} \mathrm{C}$ before processing. Plasma and tumor samples were immediately assayed for concentrations of gefitinib by HPLC as previously described (19). Tumor extracts were processed in an identical manner, except that samples were diluted using methanol:water (70:30, v/v).

Statistical analysis. The data are presented as the means \pm SEM. $\mathrm{p}<0.05$ was considered to indicate a statistically significant result. Calculation of the survival rate of the nude mice was by the Life Table method. The statistical significance was determined by the log-rank test. The statistical significance of the tumor size and weight was determined by the Student's t-test for two groups or by the Kruskal-Wallis test followed by Dunn's post-test for multiple groups. All data were analyzed using SPSS 19.0.

\section{Results}

Cellular gefitinib concentration is significantly increased in the combination group. The gefitinib concentration in the NSCLC A549 cells was detected by HPLC. Representative chromatographic profiles from the $\mathrm{G}$ and $\mathrm{TP}+\mathrm{G}$ groups are shown in Fig. 1A and B. Fig. 1C shows standard (gefitinib; $0.1 \mu \mathrm{M}$ ) chromatographic profiles; peaks at $\sim 8 \mathrm{~min}$, maximum absorption wavelength of $246 \mathrm{~nm}$. After repeating the test, the results showed that the cellular gefitinib concentration was significantly higher in the combination $(\mathrm{TP}+\mathrm{G})$ group as compared with that in the G group (Fig. 1D).

Combination drug treatment greatly inhibits tumor cell growth following the first three treatments but not after the fourth treatment. To confirm the effect of the combined treatment and the single-drug exposure, we simulated the clinical treatment model by administering gefitinib alone or in combination with TP for four times.

After the first drug treatment, cell proliferation was inhibited (Fig. 2A), the cell cycle was arrested in the G2/M phase (Fig. 2F) and marked cell death induction was observed in the $\mathrm{TP}+\mathrm{G}$ group (Fig. 2G). Compared with the normal control and TP group, the expression levels of P-EGFR, P-AKT and $\mathrm{P}-\mathrm{ERK}$ protein were decreased in the $\mathrm{G}$ and $\mathrm{TP}+\mathrm{G}$ groups. Compared with the normal control and the G group, p53 and BAX were increased in the TP and $\mathrm{TP}+\mathrm{G}$ groups (Fig. 2B). The expression of ABCG2 displayed no obvious difference among the four groups. During treatment, the $\mathrm{IC}_{50}$ values of gefitinib increased (Fig. 2E), and the cellular gefitinib concentration was always significantly higher in the combination $(\mathrm{TP}+\mathrm{G})$ when compared with that in the G group (Fig. 2D). The second and third treatment had a similar effect on cell viability in comparison to the first treatment. However, after the fourth drug treatment, cells in the TP+G group exhibited increased growth rate, reduced cell cycle arrest in the G2/M phase, and decreased apoptosis (Fig. 2F and G).

Protein levels of P-IGF-1R, P-SRC and P-ERK in the fourth $(T P+G)$ treatment group are significantly increased (Fig. 3). The expression of cell growth-associated proteins was detected after the fourth drug treatment. The levels of P-EGFR, P-IGF-1R, P-SRC and P-ERK were higher in the fourth treatment $(\mathrm{TP}+\mathrm{G}) 4$ group than these levels in the control group and the fourth G4 treatment group. The results of P-HER2, P-MET, P-PTEN are not shown due to the absence of expression in the three groups.

IGF-IR mediates drug resistance in the late combination treatment group. We aimed to determine the molecular mechanism by downregulating expression of IGF-1R and SRC in the fourth $(\mathrm{TP}+\mathrm{G}) 4$ treatment group. Results showed that cell proliferation was inhibited (Fig. 4C), and the levels of P-AKT, P-ERK, P-SRC were reduced after IGF-1R silencing with specific siRNA (Fig. 4A). Moreover, the percentage of cell apoptosis increased following exposure to gefitinib (Fig. 4B). However, all these changes were not observed after SRC was inhibited by saratinib.

$T P+G$ treatment fails in the lung cancer mouse model. The $\mathrm{TP}+\mathrm{G}$ group exhibited a significant reduction in tumor volume and mouse body weight when compared to the other groups (Fig. 5A and B). The tumor inhibitory rate (IR) in the $\mathrm{TP}+\mathrm{G}$ group was $52.7 \%$, and this rate in the $\mathrm{G}$ group was $34.28 \%$. However, the body weight of mice in the $\mathrm{TP}+\mathrm{G}$ treatment group was markedly decreased. Additionally, this group exhibited poor body condition and the shortest survival time (Fig. 5C). The concentrations of gefitinib in the serum and tumor tissues were measured by HPLC. The gefitinib concentrations in the serum and tumor tissues were higher in the gefitinib alone group when compared to the concentrations in the combination therapy group (Fig. 5D and E). The gefitinib relative absorption rate was calculated according to the following formula: Relative absorption rate $=$ (gefitinib concentration in the serum)/(gefitinib concentration in the tumor tissue). The relative absorpion rate in the $\mathrm{G}$ group was higher than that in the $\mathrm{TP}+\mathrm{G}$ group (Fig. 5F).

In the course of treatment, there was no mutation in 18, 19, 20, 21 exons of EGFR as determined by sequencing. During the treatment period, we extracted total DNA from A549, G4-A549, (TP+G)4-A549, cells designed and synthesized the primers for EGFR exons 18, 19, 20 and 21. Mutation analysis was performed by direct sequencing of the four exons. No mutation was detected throughout the treatment process.

\section{Discussion}

The present study results demonstrated that the use of a combination treatment (chemotherapy + gefitinib) resulted in a failed antitumor effect in vitro and in vivo. In the in vitro studies, the gefitinib concentration in the cells was significantly higher in the paclitaxel + cisplatin + gefitinib $(\mathrm{TP}+\mathrm{G})$ group than that in the $G$ group. Following the treatment time extension, the $\mathrm{TP}+\mathrm{G}$ treatment increased the cell viability while the cellular concentration of gefitinib was not reduced. In the A549 xenograft model, the gefitinib concentrations in the $G$ group in the serum and tumor tissues of the nude mice were higher than these levels in the combination therapy group. However, the body weight of mice in the TP+G group was markedly decreased with a poor condition and the shortest survival time. Previous studies on the combination of gefitinib and chemotherapy for initial treatment of advanced NSCLC also found the failure for an improved response or prolonged 
A

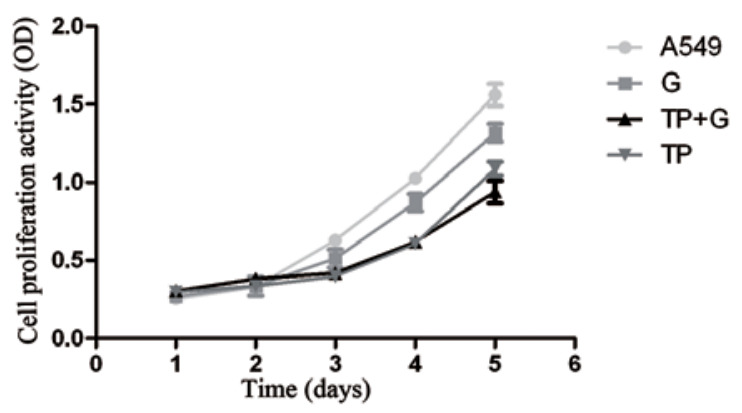

C

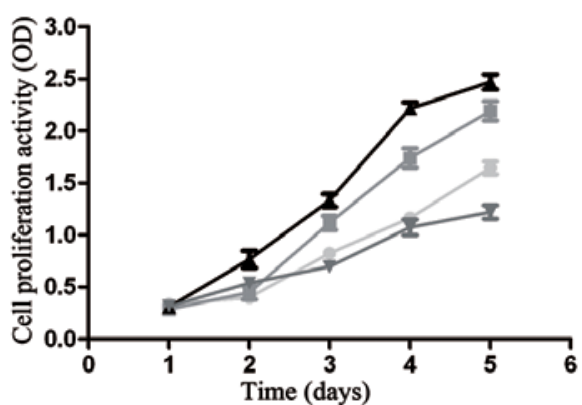

E

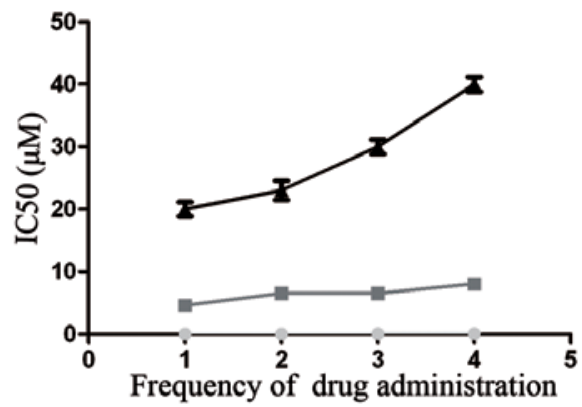

G
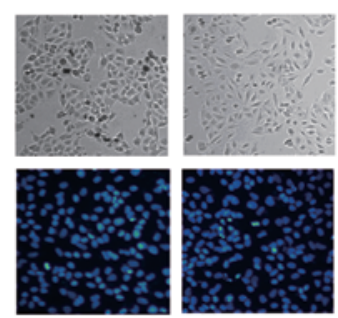

A549

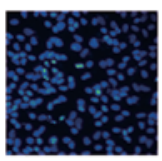

G
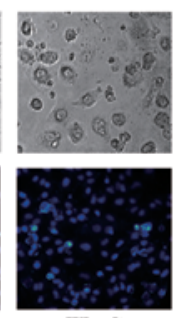

$\mathrm{TP}+\mathrm{G}$
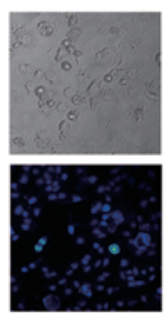

TP

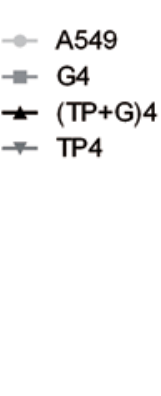

- Paclitaxel

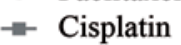

\pm Gefitinib

Figure 2. Effect of different drug treatments on NSCLC A549 cells following 4 treatments. Values are expressed as the means \pm SEM at each time-point and represent three individual experiments. (TP+G)4, the fourth TP and gefitinib treatment group. (A) CCK-8 assay of the growth-inhibitory effect of different treatments on the various groups including A549 (control), G (gefitinib), TP+G (paclitaxel, cisplatin and gefitinib) and TP. The control group exhibited the most rapid proliferation rate while the TP+G group had the slowest. (B) Different protein levels in the cells were detected by western blotting. Compared with the control and TP group, the expression levels of P-EGFR, P-AKT and P-ERK proteins were decreased in the G and TP+G groups, while p53 and BAX were increased in the TP and TP+G groups. (C) Cell proliferation was analyzed after the fourth drug treatments. After the fourth drug treatment, cells in the $(\mathrm{TP}+\mathrm{G}) 4$ group exhibited an increased growth rate $(\mathrm{p}<0.05)$. (D) Cellular gefitinib concentration was significantly higher in the TP+G group when compared with that in the $\mathrm{G}$ group $\left({ }^{*} \mathrm{p}<0.05\right)$. (E) The $\mathrm{IC}_{50}$ value of paclitaxel, cisplatin and gefitinib after each treatment in the TP+G group. After the fourth drug treatment, cells in the $(\mathrm{TP}+\mathrm{G}) 4$ group exhibited improved gefitinib $\mathrm{IC}_{50}$ values $(\mathrm{p}<0.05)$. Results are the average of duplicate experiments. (F) Cells in the TP+G group exhibited cell cycle arrest in the $\mathrm{G} 2 / \mathrm{M}$ phase ( $<0.05)$, compared with the $\mathrm{G}$ and control groups. Following the time extension, cell cycle in the fourth treatment $(\mathrm{TP}+\mathrm{G}) 4$ group was arrested in the $\mathrm{G} 0 / \mathrm{G} 1$ phase $(\mathrm{p}>0.05)$, compared with the control group. (G) Hoechst staining images of cells. The percentage of Hoechst-positive cells were counted as apoptotic. The TP+G group showed an increase in apoptosis compared with the control, $\mathrm{G}$ and TP groups $\left({ }^{*} \mathrm{p}<0.05\right)$. The fourth time $(\mathrm{TP}+\mathrm{G}) 4$ treatment group showed a decrease in the percentage of apoptotic cells when compared with the TP+G treatment group $\left({ }^{*} \mathrm{p}<0.05\right)$. 


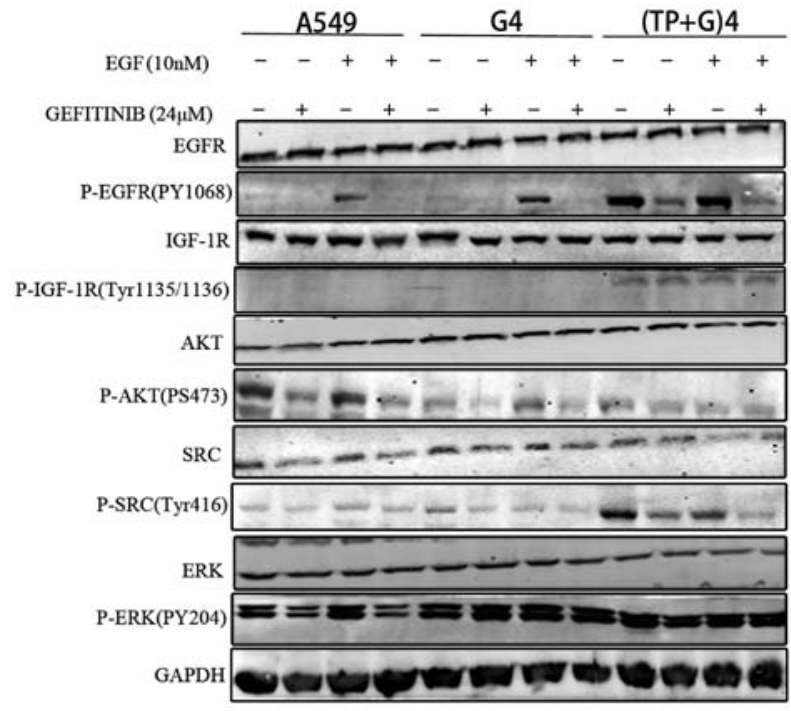

Figure 3. Protein expression levels in the cells following $10 \mathrm{nM}$ EGF or/and $24 \mu \mathrm{M}$ gefitinib stimulus for $30 \mathrm{~min}$. A549, control cells; G4, the fourth gefitinib alone treatment group; $(\mathrm{TP}+\mathrm{G}) 4$, the fourth $\mathrm{TP}$ and gefitinib treatment group. P-EGFR, P-IGF-1R, P-ERK and P-SRC levels were increased in the $(\mathrm{TP}+\mathrm{G}) 4$ group following gefitinib alone treatment. Densitometric analysis was performed, and the results were normalized to the GAPDH level. The data illustrated are representative of 3 experiments.

survival over chemotherapy alone (20). Similar results were also reported in animal models $(21,22)$. In regards to the failed combination therapy, we hypothesized that the possible reason may be that the chemotherapy drugs disturbed the cell membrane transport of gefitinib consequently resulting in failure. A previous study reported that one mechanism for resistance to EGFR-TKI is associated with the downregulation of $\mathrm{ABCG} 2$ expression (23). In the present study, the results did not demonstrate a decrease in the expression of ABCG2 following the combination therapy. Furthermore, several studies have reported that EGFR mutations or amplification can identify patients more likely to benefit or cause drug-resistance from gefitinib monotherapy $(7,24,25)$. To dismiss these confounding factors, including EGFR mutations, a high EGFR copy number, we chose the A549 cell line which is known to be $\mathrm{EFGR}^{\mathrm{WT}}$. Moreover, a fluorescent derivative of Tasigna was synthesized to study the cellular Tasigna concentration (26). However, the fluorescent derivative of Tasigna has a lower efficiency that could be attributed to the structural alteration of the molecule, resulting in decreased affinity to its target. The isotope derivative has the same problem. In this study, we established an HPLC method for determination of the content of gefitinib in the A549 cells.

In the in vivo studies, the gefitinib concentration in the gefitinib group in the serum and tumor tissues of nude mice were higher than these levels in the combination therapy group. The major human P450 enzyme involved in the metabolism of gefitinib is CYP3A4, demonstrating that CYP3A4 is primarily responsible for the overall metabolism of gefitinib. However, in the present study, there were no significant differences in CYP3A4 protein expression in the liver tissues of the nude mice. The results of such an analysis may be explained by the fact that the chemotherapy seriously affected the gastrointestinal function in the nude mice, resulting in a reduction in gefitinib absorption, which eventually led to a higher gefitinib concentration of serum and tumor tissues in the gefitinib alone group. In addition, metabolism of gefitinib was fairly rapid and complex (27). The content of gefitinib could not be detected for $>3 \mathrm{~h}$ by HPLC. Following treatment of gefitinib, the mice were sacrificed no later than $2 \mathrm{~h}$ afterwards and the gefitinib
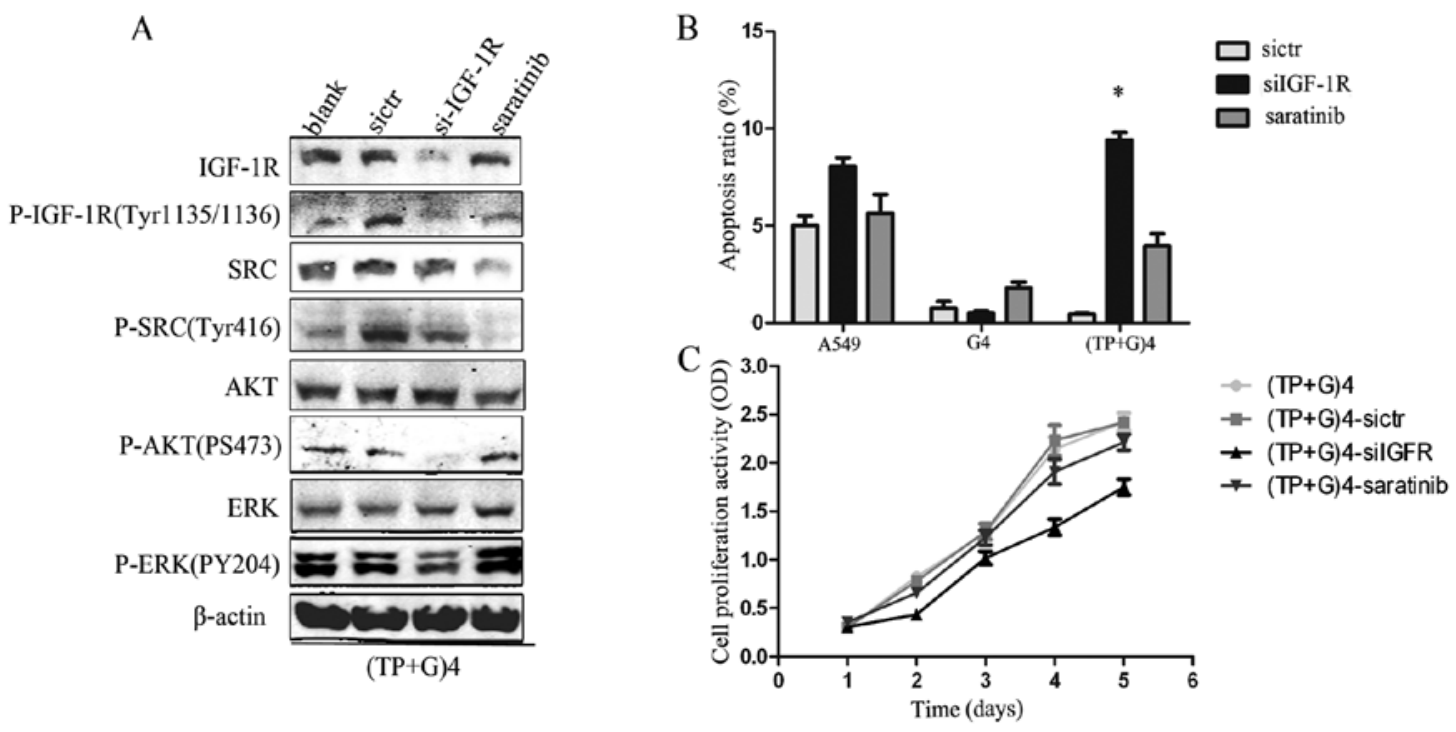

Figure 4. IGF-IR induces drug resistance in the fourth TP+G treatment group. Data represents the means \pm SEM (n=3). (A) IGF-1R and SRC expression in the $(\mathrm{TP}+\mathrm{G}) 4$ group was determined. A549 cells were transfected with sicontrol (sictr), siIGF-1R and SRC inhibitor (5 nM, saratinib). Following downregulation of IGF-1R with siRNA, the levels of P-AKT, P-ERK, P-SRC were reduced in the (TP+G)4-A549 cells. $\beta$-actin was used as a loading control. (B) A549, G4-A549 and $(\mathrm{TP}+\mathrm{G}) 4$ group cells were treated with sictr, siIGF-1R and saratinib for $48 \mathrm{~h}$, respectively. Gefitinib $(24 \mu \mathrm{M})$ was then added to continue culture for $24 \mathrm{~h}$. Cell apoptosis was increased in the (TP+G)4-siIGF-1R cells ( $\left.{ }^{*} \mathrm{p}<0.05\right)$ compared with the control and saratinib-treated cells. (C) (TP+G)4 A549 cells were separately treated by sictr, siIGF-1R and saratinib and then analyzed by CCK- 8 for cell proliferation. After downregulation of IGF-1R, cells in the (TP+G) 4 group exhibited a decreased growth rate $(\mathrm{p}<0.05)$. 
A

C

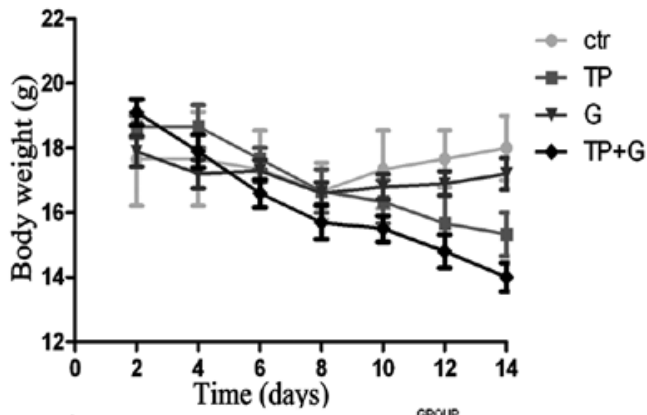

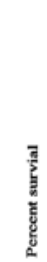

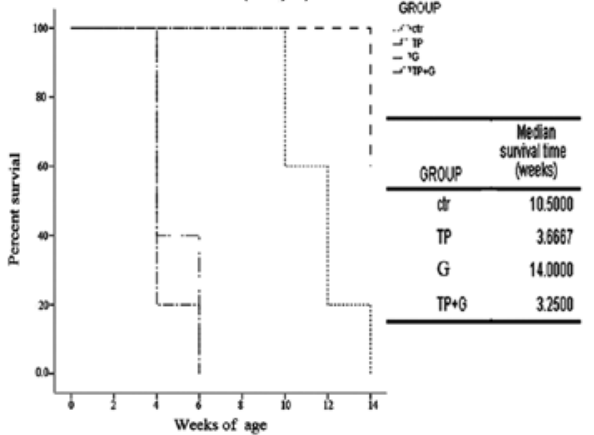

$\mathrm{E}$

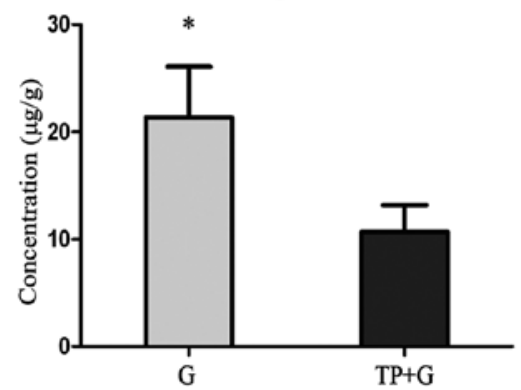

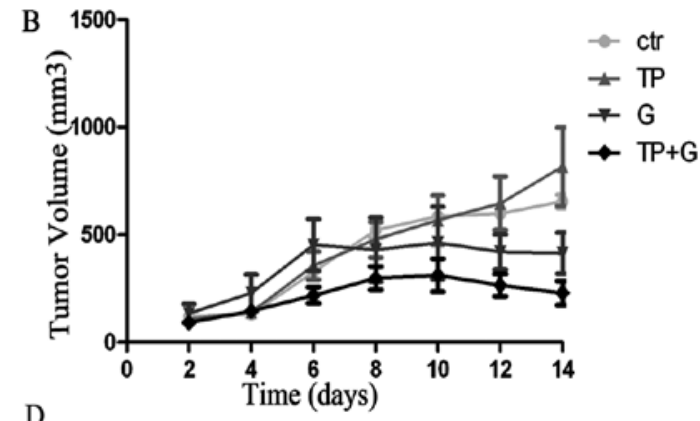

D

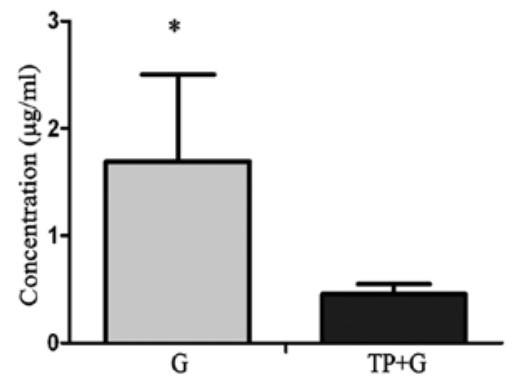

F

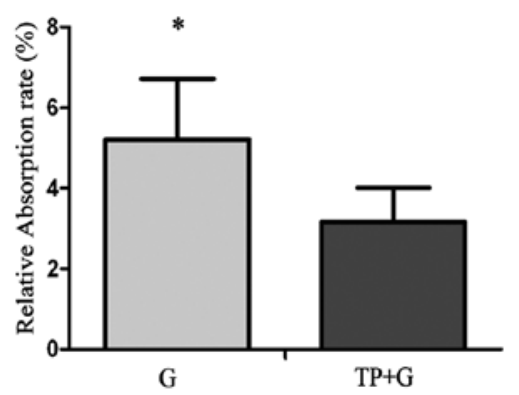

Figure 5. Failed combination treatment in a lung cancer xenograft model after 14 days of treatment. Each data value represents the mean tumor weight of 5 mice. Inhibition rate (IR) $=$ (the weight of tumors in the control group - the weight of tumors in the experimental group)/the weight of tumors in the control group $x 100 \%$. (A) The $\mathrm{TP}+\mathrm{G}$ group had the most decreased body weight compared to the control, G and $\mathrm{TP}$ groups (p<0.05). (B) $\mathrm{The} \mathrm{TP}+\mathrm{G}$ group had the smallest tumor volume compared to the control and $\mathrm{G}$ group $(\mathrm{p}<0.05)$. (C) Survival rate in the $\mathrm{G}$ group was longer than the rate in the control, TP and $\mathrm{TP}+\mathrm{G}$ groups $(\mathrm{p}<0.05)$. (D) Gefitinib concentration in the serum of the $\mathrm{G}$ group was higher than that in the $\mathrm{TP}+\mathrm{G}$ group ( $\mathrm{p}<0.05)$. (E) Gefitinib concentration in tumor tissues of the $\mathrm{G}$ group was higher than that in the $\mathrm{TP}+\mathrm{G}$ group $\left({ }^{*} \mathrm{p}<0.05\right)$. (F) Gefitinib relative absorption rate $=($ gefitinib concentration in serum)/ (gefitinib concentration in tumor tissue). Result showed that the $\mathrm{G}$ group had a higher gefitinib relative absorption rate than the $\mathrm{TP}+\mathrm{G}$ group $\left({ }^{*} \mathrm{p}<0.05\right.$ ).

concentration was immediately detected in the serum and tumor tissues. Although there are many reasons for the failed combination therapy in vivo, decreased gefitinib concentration in serum and tumor tissues could also have been a reason.

For the in vitro assay, following the first three treatments, the combination group demonstrated an obviously inhibitory effect on cell proliferation compared to gefitinib monotherapy. However, this effect was reversed after the fourth treatment. Further analysis of the protein expression found that levels of P-EGFR, P-IGF-1R, P-SRC and P-ERK were higher in the fourth $\mathrm{TP}+\mathrm{G}$ group than levels in the the fourth gefitinib alone treatment and control groups. A previous study reported that SRC can activate EGFR in colorectal cancer cell lines, and EGFR overexpression is correlated with SRC activation (28). Through transactivation, as described above, it appears that the effect of EGFR on tumorigenesis extends beyond ligandmediated effects. However, in the present study, we were unable to detect any effect of SRC. Studies have found that IGF-1R also activates P-AKT and P-ERK leading to increased cell growth. By downregulating the expression of IGF-1R in the fourth $\mathrm{TP}+\mathrm{G}$ treatment group, drug resistance was successfully reversed. Thus, we conclude that IGF-1R activation contributed to the drug resistance in the fourth $\mathrm{TP}+\mathrm{G}$ group. IGF-1R activation resulted in a marked phosphorylation level of ERK and Akt. Both of them transduce signals that trigger a cascade of cell viability responses. The signals involved in cell growth possibily reversed the effects of the high cellular concentration of gefitinib resulting in final failure of the combination treatment. IGF-1R is a transmembrane TK receptor which is activated by binding of its ligands IGF-I and IGF-II. Extensive studies have established that the receptor signaling plays an important role in tumorigenesis, metastatic potential of tumor cells and neoplastic growth (29). The consequences of IGF-1R activation by its ligands result in the recruitment of major adapter signaling proteins which lead to interaction with Grb/SOS and ultimately RAS/MAPK (ERK) signaling cascades and PI3-kinase/AKT activation (30). Evidence suggests that IGF-1R plays a role in maintaining the malignant phenotype (31), and disruption of IGF-IR activation has been shown to inhibit growth and motility of a wide range of cancer cells in vitro and in mouse models (32). In our study, we first reported that frequent use of the combined 
treatment with chemotherapy and gefitinib in the A549 cell line induced IGF-1R activation which resulted in the failed treatment. Furthermore, through paclitaxel, cisplatin, gefitinib, paclitaxel + cisplatin (TP) and (TP+G) separately stimulated, we confirmed that the treatment schedules of $\mathrm{TP}+\mathrm{G}$ caused IGF-1R activation. Although IGF-1R activation normally requires the presence of insulin-like growth factor-1, there is some precedent for IGF-1R activation without its cognate ligand (33).

In summary, the combination therapy $(\mathrm{TP}+\mathrm{G})$ appears to be ineffective for NSCLC treatment. In the in vivo assay, decreased gefitinib concentrations in the serum and tumor tissues could have been a reason for the failure of the combination therapy. More importantly, frequent use of the combination treatment in A549 lung cancer cells induced IGF-1R activation which contributed to gefitinib resistance and gave rise to the failure of the combination therapy. Although the exact molecular mechanism of IGF-1R activation remains unclear, the present study provides foundation for further study concerning the IGF activation pathway.

\section{Acknowledgements}

This study was supported by the National Natural Science Foundation of China (nos. 81071640 and 81000877), and the National Basic Research Program of China (no. 2011CB935800). The authors are grateful to all study participants.

\section{References}

1. Jemal A, Siegel R, Ward E, et al: Cancer statistics, 2008. CA Cancer J Clin 58: 71-96, 2008.

2. Mendelsohn J and Baselga J: The EGF receptor family as targets for cancer therapy. Oncogene 19: 6550-6565, 2000.

3. de Bono JS and Rowinsky EK: The ErbB receptor family: a therapeutic target for cancer. Trends Mol Med 8 (Suppl 4): S19-S26, 2002.

4. Woodburn JR: The epidermal growth factor receptor and its inhibition in cancer therapy. Pharmacol Ther 82: 241-250, 1999.

5. Gadducci A, Cosio S and Genazzani AR: Old and new perspectives in the pharmacological treatment of advanced or recurrent endometrial cancer: hormonal therapy, chemotherapy and molecularly targeted therapies. Crit Rev Oncol Hematol 58: 242-256, 2006.

6. Nakagawa K, Tamura T, Negoro S, et al: Phase I pharmacokinetic trial of the selective oral epidermal growth factor receptor tyrosine kinase inhibitor gefitinib ('Iressa', ZD1839) in Japanese patients with solid malignant tumors. Ann Oncol 14: 922-930, 2003.

7. Fukuoka M, Yano S, Giaccone G, et al: Multi-institutional randomized phase II trial of gefitinib for previously treated patients with advanced non-small-cell lung cancer. J Clin Oncol 21: 2237-2246, 2003.

8. Natale R, Skarin A, Maddox A, et al: Improvement in symptoms and quality of life for advanced non-small-cell lung cancer patients receiving ZD1839 ('Iressa') in IDEAL 2. Proc Am Soc Clin Oncol 21: 292a, 2002.

9. Rivera F, Vega-Villegas ME and López-Brea MF: Cetuximab, its clinical use and future perspectives. Anticancer Drugs 19: 99-113, 2008.

10. Pirker R, Pereira JR, Szczesna A, et al: Cetuximab plus chemotherapy in patients with advanced non-small-cell lung cancer (FLEX): an open-label randomised phase III trial. Lancet 373: 1525-1531, 2009.

11. Kris MG, Natale RB, Herbst RS, et al: Efficacy of gefitinib, an inhibitor of the epidermal growth factor receptor tyrosine kinase, in symptomatic patients with non-small cell lung cancer: a randomized trial. JAMA 290: 2149-2158, 2003.
12. Franceschi E, Cavallo G, Lonardi S, et al: Gefitinib in patients with progressive high-grade gliomas: a multicentre phase II study by Gruppo Italiano Cooperativo di Neuro-Oncologia (GICNO). Br J Cancer 96: 1047-1051, 2007.

13. Salzberg M, Rochlitz C, Morant R, et al: An open-label, noncomparative phase II trial to evaluate the efficacy and safety of docetaxel in combination with gefitinib in patients with hormone-refractory metastatic prostate cancer. Onkologie 30: 355-360, 2007.

14. Small EJ, Fontana J, Tannir N, et al: A phase II trial of gefitinib in patients with non-metastatic hormone-refractory prostate cancer. BJU Int 100: 765-769, 2007.

15. Goncalves A, Fabbro M, Lhommé C, et al: A phase II trial to evaluate gefitinib as second- or third-line treatment in patients with recurring locoregionally advanced or metastatic cervical cancer. Gynecol Oncol 108: 42-46, 2008.

16. Giaccone G, González-Larriba J, Van Oosterom A, et al: Combination therapy with gefitinib, an epidermal growth factor receptor tyrosine kinase inhibitor, gemcitabine and cisplatin in patients with advanced solid tumors. Ann Oncol 15: 831-838, 2004.

17. Giaccone G, Herbst RS, Manegold C, et al: Gefitinib in combination with gemcitabine and cisplatin in advanced non-small-cell lung cancer: a phase III trial - INTACT 1. J Clin Oncol 22: 777-784, 2004.

18. Breathnach OS, Freidlin B, Conley B, et al: Twenty-two years of phase III trials for patients with advanced non-small-cell lung cancer: sobering results. J Clin Oncol 19: 1734-1742, 2001.

19. McKillop D, Partridge EA, Hutchison M, et al: Pharmacokinetics of gefitinib, an epidermal growth factor receptor tyrosine kinase inhibitor, in rat and dog. Xenobiotica 34: 901-915, 2004.

20. Herbst RS, Giaccone G, Schiller JH, et al: Gefitinib in combination with paclitaxel and carboplatin in advanced non-small-cell lung cancer: a phase III trial - INTACT 2. J Clin Oncol 22: 785-794, 2004.

21. Ciardiello F, Caputo R, Bianco R, et al: Antitumor effect and potentiation of cytotoxic drugs activity in human cancer cells by ZD-1839 (Iressa), an epidermal growth factor receptor-selective tyrosine kinase inhibitor. Clin Cancer Res 6: 2053-2063, 2000.

22. Sirotnak FM, Zakowski MF, Miller VA, Scher HI and Kris MG: Efficacy of cytotoxic agents against human tumor xenografts is markedly enhanced by coadministration of ZD1839 (Iressa), an inhibitor of EGFR tyrosine kinase. Clin Cancer Res 6: 4885-4892, 2000.

23. Ohtsuka K, Ohnishi H, Morii T, et al: Downregulated ABCG2 enhances sensitivity to topoisomerase I inhibitor in epidermal growth factor receptor tyrosine kinase inhibitor-resistant non-small cell lung cancer. J Thorac Oncol 5: 1726-1733, 2010.

24. Cohen MH, Williams GA, Sridhara R, Chen G and Pazdur R: FDA drug approval summary: gefitinib (ZD1839) (Iressa ${ }^{\circledR}$ ) tablets. Oncologist 8: 303-306, 2003.

25. Miller VA, Kris MG, Shah N, et al: Bronchioloalveolar pathologic subtype and smoking history predict sensitivity to gefitinib in advanced non-small-cell lung cancer. J Clin Oncol 22: 1103-1109, 2004.

26. Shukla S, Skoumbourdis AP, Walsh MJ, et al: Synthesis and characterization of a BODIPY conjugate of the BCR-ABL kinase inhibitor Tasigna (nilotinib): evidence for transport of Tasigna and its fluorescent derivative by $\mathrm{ABC}$ drug transporters. Mol Pharm 8: 1292-1302, 2011.

27. McKillop D, McCormick A, Miles GS, et al: In vitro metabolism of gefitinib in human liver microsomes. Xenobiotica 34: 983-1000, 2004.

28. Osherov N and Levitzki A: Epidermal-growth-factor-dependent activation of the src-family kinases. Eur J Biochem 225: 1047-1053, 1994.

29. Pollak M, Beamer W and Zhang JC: Insulin-like growth factors and prostate cancer. Cancer Metastasis Rev 17: 383-390, 1998.

30. Sachdev D and Yee D: The IGF system and breast cancer. Endocr Relat Cancer 8: 197-209, 2001.

31. Pollak MN, Schernhammer ES and Hankinson SE: Insulin-like growth factors and neoplasia. Nat Rev Cancer 4: 505-518, 2004.

32. Baserga R: Targeting the IGF-1 receptor: from rags to riches. Eur J Cancer 40: 2013-2015, 2004.

33. Frattali A and Pessin J: Relationship between $\alpha$ subunit ligand occupancy and $\beta$ subunit autophosphorylation in insulin/insulinlike growth factor-1 hybrid receptors. J Biol Chem 268: 7393-7400, 1993. 